\title{
Induction of Necrotizing Funisitis by Fetal Administration of Intravenous Granulocyte-Colony Stimulating Factor and Intra-Amniotic Endotoxin in Premature Fetal Sheep
}

\author{
TATSUYA WATANABE, TADASHI MATSUDA, TAKUSHI HANITA, KAZUHIKO OKUYAMA, KAZUTOSHI CHO, \\ KUNIHIKO KOBAYASHI, AND YOSHIYASU KOBAYASHI
}

\begin{abstract}
Department of Pediatrics [T.W., T.M., T.H.], Tohoku University Graduate School of Medicine, Sendai 980-8574, Japan; Department of Obstetrics and Gynecology [K.O.], Department of Pediatrics [K.C., K.K.], Hokkaido University Graduate School of Medicine, Sapporo 060-8648, Japan; Department of Veterinary Pathology [Y.K.], Obihiro University of Agriculture and Veterinary Medicine, Obihiro 080-8555, Japan
\end{abstract}

\begin{abstract}
The purpose of the present study was to determine whether experimental intrauterine inflammation could induce necrotizing funisitis, a severe, chronic inflammation of the umbilical cord. Fetuses, randomly divided into four groups ( $n=4$ each), were infused with $50 \mu \mathrm{g} / \mathrm{d}$ of granulocyte-colony stimulating factor (G-CSF) intravenously on d 125-129 of gestation (G-CSF group), $20 \mathrm{mg}$ of endotoxin into the amniotic cavity on d 127 gestation (endotoxin group), both G-CSF and endotoxin (G-CSF + endotoxin group), or only saline (control group). On d 130 of gestation, the umbilical cords were processed for histologic analysis, scored for degree of inflammation, and compared statistically. At birth, the blood polymorphonuclear leukocyte counts in G-CSF and G-CSF + endotoxin groups were significantly higher than those in endotoxin and control groups $(p<0.05)$. The inflammatory score of the umbilical cord in G-CSF + endotoxin group was significantly higher than those in the other three groups $(p<0.05)$. All the fetuses in G-CSF + endotoxin group had necrotizing funisitis, but none of the fetuses in the other three groups developed this condition. An increase in blood polymorphonuclear leukocytes before their activation in the umbilical cord is probably essential for experimentally inducing necrotizing funisitis. (Pediatr Res 62: 670-673, 2007)
\end{abstract}

A though intrauterine inflammation is well known as a major cause of preterm labor and premature rupture of the fetal membranes (1), recent reports have also noted a relation to the development of cerebral white matter injuries or chronic lung disease (CLD), which are serious risk factors for adverse outcomes in premature infants (2). Clinical and experimental studies suggest that inflammation in utero, such as chorioamnionitis and funisitis, directly affects premature fetal organs and their development in a manner that is independent from prematurity (3-6). However, the pathophysiological involvement of intrauterine inflammation in the genesis of fetal organ injuries has not been fully clarified.

Received March 26, 2007; accepted June 28, 2007

Correspondence: Tadashi Matsuda, M.D., Department of Pediatrics, Tohoku University Graduate School of Medicine, 1-1, Seiryo-machi, Aoba-ku, Sendai 980-8574, Japan; e-mail: choku@mail.tains.tohoku.ac.jp

Support provided by the Ogyaa Donation Foundation of the Japan Association of Obstetricians and Gynecologists and a Grant-in-Aid for Scientific Research (No. 13671686) from the Ministry of Education, Culture, Sports, Science and Technology in Japan.
Necrotizing funisitis is a chronic and severe inflammation of the umbilical cord in which numerous neutrophils migrate and infiltrate from the vascular space toward the amniotic cavity and old necrotic exudate is concentrically deposited in a ring-like fashion around the vessels, frequently with calcification (7-9). We previously reported that necrotizing funisitis was significantly associated with the development and exacerbation of CLD in premature infants and indicated that an increased polymorphonuclear leukocyte (PMNL) count in the fetal blood and the existence of some inflammatory inducer in the amniotic fluid appeared to have important roles in the genesis of CLD (10). To analyze the antenatal effects of inflammatory intensity or duration on fetal lung injury, however, an experimental animal model of necrotizing funisitis was needed. If such a model could be used to improve our understanding of these effects, the development of preventive and therapeutic measures for CLD in premature infants might be advanced.

The aim of the current study was to develop an experimental animal model of necrotizing funisitis using chronically instrumented fetal sheep. To induce a more exacerbated inflammation in utero than that observed in previous experiments (11-13), the fetuses were intravenously treated with granulocyte-colony stimulating factor (G-CSF) to increase their blood PMNL counts before endotoxin infusion into the amniotic cavity, thereby activating the PMNL in utero.

\section{METHODS}

\begin{abstract}
Animal preparation. With the approval of the Animal Care and Use Committee of the Hokkaido (No. 2058) and the Tohoku (No. 15-128) University School of Medicine, this study was carried out from April 2001 to April 2004. The preparations for this experiment were mostly the same as those for our previous studies (14). In brief, a total of 16 Suffolk ewes with timed pregnancies underwent surgery on d 123 of gestation. The ewes were anesthetized with intrathecal tetracaine hydrochloride and intravenous ketamine hydrochloride during all the procedures. After a laparotomy and hysterotomy, polyvinyl catheters were placed into the fetal carotid artery and vein and into the amniotic cavity. All catheters were exteriorized through a small incision in the flank of each ewe. After surgery, the ewes were unrestrained and housed in individual cages, with free access to water and
\end{abstract}

Abbreviations: CLD, chronic lung disease; G-CSF, granulocyte-colony stimulating factor; PMNL, polymorphonuclear leukocyte 
food throughout the study period. A recovery period of at least $2 \mathrm{~d}$ was allowed before starting the experiments; during that time, antibiotics were administered to the mother, fetus, and amniotic cavity based on the results of Gram's stain tests using amniotic fluid smears.

Experimental protocol. At least $48 \mathrm{~h}$ after surgery on d 125 of gestation, the fetuses were randomly divided into four groups. The fetuses in the G-CSF group $(n=4)$ were given a bolus infusion of $50 \mu \mathrm{g}$ of G-CSF (Neutrogin R; Chugai Co. Ltd, Tokyo, Japan) solublized in $2 \mathrm{~mL}$ of saline into the carotid vein daily from d 125 to $\mathrm{d} 129$ of gestation. Fetuses in the endotoxin group $(n=4)$ were injected with $20 \mathrm{mg}$ of endotoxin (Escherichia coli 055:B5 endotoxin; Sigma Chemical Co., St. Louis, MO) solubilized in $2 \mathrm{~mL}$ of saline into the amniotic cavity once on d 127 of gestation. Fetuses in the G-CSF + endotoxin group $(n=4)$ were treated with both G-CSF and endotoxin in the manners described above, whereas those in the control group $(n=4)$ were treated with only saline.

Histopathologic examination. On d 130 of gestation, cesarean sections were performed and the fetuses were weighed. The umbilical cord, roll of membrane (amnion and chorion), and placentomes were placed in $10 \%$ neutralized buffered formalin for fixation. Histopathologic evaluation was performed using $4-\mu \mathrm{m}$ sections stained with hematoxylin and eosin. The influx of inflammatory cells into the tissues was graded by scoring the total fields of view in more than three sections and applying the inflammatory grading system reported by Salafia et al. (15), to which we added necrotizing funisitis defined using the criteria of Navarro and Blanc (8) (Table 1). All assessments were made by the same observer (Y.K.) in a blinded fashion.

Measurements of physiologic parameters. Fetal heart rates and arterial and amniotic pressures were continuously monitored using a polygraph and recorded on a personal computer throughout the study. All fetal arterial pressure values were corrected for the amniotic fluid pressure. The total leukocyte counts (Celtac MEK-5254; Nihon-Koden Co, Tokyo, Japan) and $\mathrm{pH}$, base excess, $\mathrm{PCO}_{2}$, and Po 2 (Blood Gas System 860; Bayer Medical Co., Sudbury, UK) were measured in blood samples $(0.5 \mathrm{~mL})$ taken from the fetal carotid artery every $12 \mathrm{~h}$ during the experiment. PMNLs were identified using differential staining (May-Giemsa) and counted microscopically relative to the total leukocyte count. The blood gas data were corrected using the maternal rectal temperature.

Statistical analysis. All values were expressed as the mean \pm SEM. The fetal body weight, PMNL counts, and histologic inflammatory scores among the four groups were statistically compared using the two-way Kruskal-Wallis test; if a significant difference was suggested, the Scheffé test was performed for multiple comparisons between the groups. Changes in the PMNL counts over time within each group were assessed using the Dunnett test. A $p<0.05$ was considered to be statistically significant.

\section{RESULTS}

Continuous monitoring of all the parameters showed that all 16 fetuses were in good condition; there were no occasions when the mean blood pressure was under $35 \mathrm{~mm} \mathrm{Hg}$ or the heart rate was less than 100 beats per minute over $3 \mathrm{~min}$ during the study period. Fetal $\mathrm{pH}$ was never under 7.25, and the $\mathrm{Po}_{2}$ was never less than $10 \mathrm{~mm} \mathrm{Hg}$. Neither intrauterine infection nor threatened preterm labor occurred. All of the

Table 1. Inflammatory scoring system for umbilical cord and fetal membrane

\footnotetext{
Scores for umbilical cord

$0=$ No focus of PMNL in the umbilical cord

$1=$ PMNL within the inner third of the umbilical vein wall

$2=$ PMNL within the inner third of at least two umbilical vessel walls

$3=$ PMNL in the perivascular Wharton's jelly

4 = Panvasculitis and funisitis extending deep into the Wharton's jelly

$5=$ Necrotizing funisitis

Scores for fetal membranes (amnion and chorion-decidua)

$0=$ No focus of PMNL in the amnion and chorion-decidua

$1=$ One focus of at least five PMNLs

$2=$ More than one focus of score 1 inflammation or one focus of five to 20 PMNLs

$3=$ Multiple and/or confluent foci of score 2

$4=$ Diffuse and dense acute inflammation

$5=$ Subacute or chronic inflammation with necrotic changes
}

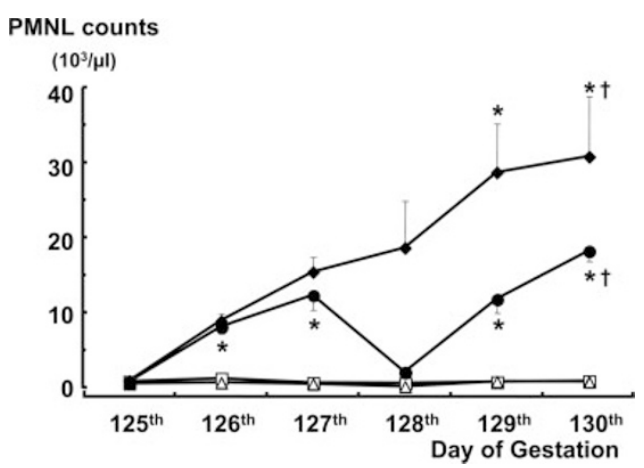

Figure 1. Changes in PMNL counts in fetal carotid artery blood during the study period in the four groups. The fetuses in the G-CSF group $(\diamond, n=4)$ were infused with $50 \mu \mathrm{g}$ of G-CSF daily from d 125 to d 129 of gestation. Fetuses in the endotoxin group $(\Delta, n=4)$ were injected with $20 \mathrm{mg}$ of endotoxin into the amniotic cavity once on d 127 of gestation. Fetuses in the G-CSF + endotoxin group $(\bullet, n=4)$ were treated with both G-CSF and endotoxin in the same manners described above, whereas those in the control group ( $\square, n=4)$ were treated with only saline. All data are expressed as the mean \pm SEM. $* p<0.05$ (Dunnett test), compared with the value on $\mathrm{d} 125$ of gestation in each group. $\dagger p<0.05$ (Scheffé test), compared with the values of the control and endotoxin groups on d 130 of gestation.

umbilical cords, placentomes, and fetal membranes were available for pathologic examination.

No significant difference in the body weight at birth was seen among the four groups (control group, $2.99 \pm 0.48 \mathrm{~kg}$; G-CSF group, $3.49 \pm 0.27 \mathrm{~kg}$; endotoxin group, $4.20 \pm 0.50$ $\mathrm{kg}$; G-CSF + endotoxin group, $3.50 \pm 0.45 \mathrm{~kg}$ ).

Figure 1 shows the changes in fetal PMNL counts over time in the four groups during the study period. The PMNL counts in the G-CSF group continuously increased and, after d 129 of gestation, reached significantly higher values compared with those measured before the G-CSF administration $(p<0.05)$. While those in the G-CSF + endotoxin group also significantly increased after d 126 of gestation, but then transiently decreased for $1 \mathrm{~d}$ immediately after the endotoxin injection on d 127 of gestation and then resumed increasing. No significant changes in the fetal PMNL counts over time were observed in the endotoxin and control groups. At birth, the PMNL counts in the G-CSF $\left(30.8 \pm 8.0 \times 10^{3} / \mu \mathrm{L}\right)$ and the G-CSF + endotoxin $\left(18.2 \pm 1.4 \times 10^{3} / \mu \mathrm{L}\right)$ groups were significantly higher than those in the endotoxin $\left(0.8 \pm 0.3 \times 10^{3} / \mu \mathrm{L}\right)$ and control groups $\left(0.9 \pm 0.3 \times 10^{3} / \mu \mathrm{L}\right)$, respectively $(p<0.05)$.

Histopathologic examinations of the umbilical cords demonstrated that all of the fetuses in the G-CSF + endotoxin group exhibited necrotizing funisitis, the infiltration of numerous PMNLs from the vascular space toward the amniotic cavity, degeneration, and the accumulation of basophilic necrotic debris in the form of ring-like or crescent-shaped bands around the umbilical vessels (Fig. 2, $A$ and $B$ ). These characteristics were not observed in the other three groups. The umbilical cords in the endotoxin group had moderate vasculitis but did not show necrotic changes, while those in the G-CSF and control groups exhibited little infiltration of PMNLs in the vascular wall. In the fetal membranes, severe chorioamnionitis with necrotic changes (Fig. 2C) was found in two of the four fetuses in the endotoxin group and three of the four fetuses in the G-CSF + endotoxin group, whereas only 

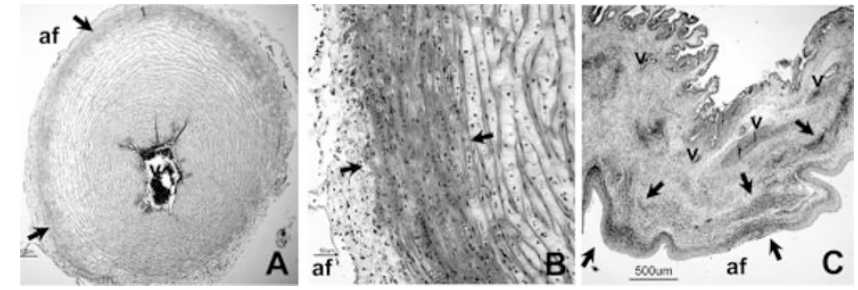

Figure 2. Hematoxylin and eosin-stained sections in the G-CSF + endotoxin group showing necrotizing funisitis $(A$ and $B)$ and chorioamnionitis $(C)$. A large degree of PMNL infiltration from the vascular space (v) toward the amniotic cavity (af ) and numerous degenerating neutrophils and abundant basophilic debris (arrows), accumulated in the form of ring-like bands around the umbilical artery $(A$ and $B$ ) and within the epithelial layer in the membrane $(C)$ are visible. ( $A$ and $B$ : short-axis cross-section of the umbilical cord, original magnification, $A: \times 40 ; B: \times 200$. C: cross-section of fetal membrane, original magnification $\times 200$.)

mild infiltration of PMNLs was found in the other two groups. PMNL infiltration or degenerative changes were not found in the fetal cotyledon and interdigitated villi of any of the placentomes in all four groups.

Table 2 shows the results of the statistical analysis of the inflammatory scores of the umbilical cords and fetal membranes in the four groups. The inflammatory scores of the umbilical cords in the G-CSF + endotoxin group were significantly higher than those in the other three groups $(p<$ $0.05)$. The inflammatory scores of the fetal membranes in the endotoxin and G-CSF + endotoxin groups were significantly higher than those in the control and G-CSF groups $(p<0.05)$, whereas no significant difference in the inflammatory score was observed between the endotoxin and G-CSF + endotoxin groups.

\section{DISCUSSION}

In the current experiment, fetuses were treated with G-CSF to increase their blood PMNL counts before activation in utero by an intra-amniotic infusion of endotoxin to induce necrotizing funisitis, a chronic and severe inflammation of the umbilical cord. The G-CSF pretreatment was thought to be necessary because the development of necrotizing funisitis may be associated with an increased G-CSF level in the fetal blood, though this has not yet been proven in a clinical study. Premature infants who suffered from chorioamnionitis were reported to have significantly higher G-CSF levels in their umbilical cord blood than those without chorioamnionitis (16). In addition, as we have reported, premature infants who had chorioamnionitis with necrotizing funisitis at birth had significantly higher blood PMNL counts than those without this condition (10).

The blood PMNL counts in the G-CSF and G-CSF + endotoxin groups were significantly higher, as expected (Fig. 1). The transient decrease in the blood PMNL counts in the G-CSF + endotoxin group just after the endotoxin infusion probably reflected the regional migration and infiltration of massive numbers of activated PMNLs from the vascular space toward the amniotic cavity in the fetal membrane and the umbilical cord, as previously reported in experiments on intra-amniotic endotoxin infusion in pregnant sheep $(12,13)$.

Histopathologically, necrotic changes in the umbilical cord were observed only in all of the fetuses in the G-CSF + endotoxin group, in which degenerated PMNLs and necrotic debris were concentrically accumulated in the form of ring-like or crescent-like bands around the vessels (Fig. $2, A$ and $B$ ). The characteristics of these changes were quite similar to necrotizing funisitis, though no calcification along the necrotic lesion was found. The quick necrotizing response after endotoxin exposure for $3 \mathrm{~d}$ was interesting, though the duration might be too short to have induced calcified changes in the umbilical tissue. The fetuses in the endotoxin group exhibited acute funisitis to some degree, but necrotic changes in the umbilical tissue were not observed, and none of the fetuses in the G-CSF group had funisitis, whereas necrotic changes in the fetal membrane were observed both in the endotoxin and G-CSF + endotoxin groups (Table 2). Therefore, the experimental induction of necrotizing funisitis was thought to require an increase in fetal blood PMNLs before their activation in utero by the intra-amniotic infusion of endotoxin, though in the fetal membrane, the intra-amniotic endotoxin was capable of inducing necrotic inflammation without an increase in blood PMNLs.

Differences in the anatomic or histologic structures of the fetal membranes and the umbilical cord might explain the importance of an increase in blood PMNLs for the induction of necrotizing funisitis. First, the vasculitis in the umbilical cord may have been more difficult to extend and accelerate in Wharton's jelly than in the fetal membranes because Wharton's jelly, surprisingly, has fewer macrophages than amniotic, chorionic, and decidual tissues (17). Second, PMNLs in the umbilical cord would move faster than those in the fetal membrane because Wharton's jelly contains only two main

Table 2. Comparison of inflammatory scores of umbilical cords and fetal membranes in the control, G-CSF, endotoxin, and G-CSF + endotoxin groups

\begin{tabular}{lcccc}
\hline & $\begin{array}{c}\text { Control group } \\
(n=4)\end{array}$ & $\begin{array}{c}\text { G-CSF group } \\
(n=4)\end{array}$ & $\begin{array}{c}\text { Endotoxin group } \\
(n=4)\end{array}$ & $\begin{array}{c}\text { G-CSF + endotoxin group } \\
(n=4)\end{array}$ \\
\hline Umbilical cord & $0.3 \pm 0.3$ & $0.0 \pm 0.0$ & $3.5 \pm 0.3$ & $5.0 \pm 0.0^{*}$ \\
Fetal membrane & $1.5 \pm 0.6$ & $2.3 \pm 0.5$ & $4.5 \pm 0.3^{* *}$ & $4.8 \pm 0.3^{* *}$ \\
\hline
\end{tabular}

All data are expressed as themean \pm SEM.

$* p<0.05$, compared with the values of the control, G-CSF, and endotoxin groups (Scheffé test).

** $p<0.05$, compared with the values of the control and G-CSF groups (Scheffé test). 
arteries and two veins, but no blood or lymphatic capillaries (9). Consequently, the PMNLs in the umbilical cord might have fewer chances or a shorter time during which they can be stimulated by the intra-amniotic endotoxin, compared with those in the fetal membranes. In this context, the increase in blood PMNL in the G-CSF + endotoxin group probably compensated for the weak and short period of stimulation by the activated macrophages, thereby exacerbating the inflammation in the Wharton's jelly.

This study demonstrated a clear causative role of an increase in blood PMNL before intra-amniotic endotoxin infusion in the genesis of necrotizing funisitis in premature fetal sheep. This experimental animal model is thought to correspond to clinical chorioamnionitis with necrotizing funisitis. Because few reports have analyzed the effects of the intensity and duration of intrauterine inflammation on the development of fetal organ injuries, such as cerebral white matter injuries (18) and CLD $(19,20)$, our experimental system is quite promising in terms of understanding the effects of exacerbating inflammation in utero on fetal cerebral hemodynamics, the premature oligodendrocytes system, and the lung surfactant system and alveolarization. Such studies are now in progress in our laboratory.

Acknowledgments. The authors thank Satoru Okajima, M.D., and Keiko Ueda, M.D., for their technical assistance.

\section{REFERENCES}

1. Goldenberg RL, Hauth JC, Andrews WW 2000 Intrauterine infection and preterm delivery. N Engl J Med 342:1500-1507

2. Gomez R, Romero R, Ghezzi F, Yoon BH, Mazor M, Berry SM 1998 The fetal inflammatory response syndrome. Am J Obstet Gynecol 179:194-202

3. Volpe JJ 2001 Neurobiology of periventricular leukomalacia in the premature infant. Pediatr Res 50:553-562
4. Duncan JR, Cock ML, Suzuki K, Scheerlink JY, Harding R, Rees SM 2006 Chronic endotoxin exposure causes brain injury in the ovine fetus in the absence of hypoxemia. J Soc Gynecol Investig 13:87-96

5. Yoon BH, Romero R, Kim KS, Park JS, Ki SH, Kim BI, Jun JK 1999 A systemic fetal inflammatory response and the development of bronchopulmonary dysplasia. Am J Obstet Gynecol 181:773-779

6. Ueda K, Cho K, Matsuda T, Okajima S, Uchida M, Kobayashi Y, Minakami H, Kobayashi K 2006 A rat model for arrest of alveolarization induced by antenatal endotoxin administration. Pediatr Res 59:396-400

7. Benirschke K, Kaufmann P 1995 Infectious disease, necrotizing funisitis. In Benirschke K, Kaufmann P (eds) Pathology of the Human Placenta, 3rd Ed. Springer-Verlag, New York, pp 568-571

8. Navarro C, Blanc WA 1974 Subacute necrotizing funisitis. J Pediatr 85:689-697

9. Craver RD, Baldwin VJ 1992 Necrotizing funisitis. Obstet Gynecol 79:64-70

10. Matsuda T, Nakajima T, Hattori S, Hanatani K, Fukazawa Y, Kobayashi K, Fujimoto S 1997 Necrotizing funisitis: clinical significance and association with chronic lung disease in premature infants. Am J Obstet Gynecol 177:1402-1407

11. Jobe AH, Newnham JP, Willet KE, Sly P, Ervin MG, Bachurski C, Possmayer F, Hallman M, Ikegami M 2000 Effects of antenatal endotoxin and glucocorticoids on the lungs of preterm lambs. Am J Obstet Gynecol 182:401-408

12. Kallapur SG, Willet KE, Jobe AH, Ikegami M, Bachurski CJ 2001 Intra-amniotic endotoxin: chorioamnionitis precedes lung maturation in preterm lambs. Am J Physiol Lung Cell Mol Physiol 280:L527-L536

13. Nitsos I, Moss TJ, Cock ML, Harding R, Newnham JP 2002 Fetal response to intra-amniotic endotoxin in sheep. J Soc Gynecol Investig 9:80-85

14. Matsuda T, Okuyama K, Cho K, Okajima S, Kobayashi Y, Hoshi Y, Kobayashi K 2006 Cerebral hemodynamics during the induction of antenatal periventricular leukomalacia by hemorrhagic hypotension in chronically instrumented fetal sheep. Am J Obstet Gynecol 194:1057-1063

15. Salafia CM, Weigl C, Silberman L 1989 The prevalence and distribution of acute placental inflammation in uncomplicated term pregnancies. Obstet Gynecol 73:383389

16. Li Y, Ohls RK, Rosa C, Shah M, Richards DS, Christensen RD 1995 Maternal and umbilical serum concentrations of granulocyte colony-stimulating factor and its messenger RNA during clinical chorioamnionitis. Obstet Gynecol 86:428-432

17. Benirschke K, Kaufmann P 1995 Anatomy and pathology of the umbilical cord and major fetal vessels, Wharton's jelly. In: Benirschke K, Kaufmann P (eds) Pathology of the Human Placenta, 3rd Ed. Springer-Verlag, New York, pp 321

18. Nitsos I, Rees SM, Duncan J, Kramer BW, Harding R, Newnham JP, Moss TJ 2006 Chronic exposure to intra-amniotic lipopolysaccharide affects the ovine fetal brain. J Soc Gynecol Investig 13:239-247

19. Kramer BW, Moss TJ, Willet KE, Newnham JP, Sly PD, Kallapur SG, Ikegami M, Jobe AH 2001 Dose and time response after intraamniotic endotoxin in preterm lambs. Am J Respir Crit Care Med 164:982-988

20. Kallapur SG, Nitsos I, Mioss TJ, Kramer BW, Newnham JP, Ikegami M, Jobe AH 2005 Chronic endotoxin exposure does not cause sustained structural abnormalities in the fetal sheep lungs. Am J Physiol Lung Cell Mol Physiol 288:L966-L974 